\title{
Safety of Chloroquine or Hydroxychloroquine Plus Azithromycin for the Treatment of COVID-19 Patients in Burkina Faso: An Observational Prospective Cohort Study
}

\author{
Toussaint Rouamba (D) ${ }^{1, *}$ \\ Houreratou Barry (iD) ${ }^{2, *}$ \\ Esperance Ouédraogo' \\ Marc Christian Tahita' \\ Nobila Valentin Yaméogo ${ }^{3}$ \\ Armel Poda ${ }^{4}$ \\ Arnaud Eric Diendéré ${ }^{5}$ \\ Abdoul-Salam Ouedraogo (D) ${ }^{4}$ \\ Innocent Valea (iD)' \\ Amariane M Koné ${ }^{2}$ \\ Cherileila Thiombiano ${ }^{2}$ \\ Isidore Traoré ${ }^{2}$ \\ Zekiba Tarnagda' \\ Serge A Sawadogo 6 \\ Zakaria Gansané ${ }^{7}$ \\ Yibar Kambiré (DD ${ }^{8}$ \\ Idrissa Sanou ${ }^{8}$ \\ Fatou Barro-Traoré ${ }^{8}$ \\ Maxime K Drabo' \\ Halidou Tinto (iD) \\ On behalf of the CHLORAZ Study \\ Group
}

'Institut de Recherche en Sciences de la Santé (CNRSTIRSS), Nanoro, Burkina Faso; ${ }^{2}$ Institut National de Santé Publique, Centre Muraz, Bobo-Dioulasso, Burkina Faso; ${ }^{3}$ Centre Hospitalier Universitaire Yalgado Ouédraogo, Ouagadougou, Burkina Faso; ${ }^{4}$ Centre Hospitalier Universitaire Sourou Sanon, Bobo-Dioulasso, Burkina Faso; ${ }^{5}$ Centre Hospitalier Universitaire de Bogodogo, Ouagadougou, Burkina Faso; ${ }^{6}$ Centre PrïmO Nelson Mandela (Promotion de la Recherche et de l'Innovation en Immunologie Médicale de Ouagadougou), Ouagadougou, Burkina Faso; ${ }^{7}$ Clinical Monitoring in Africa-Clinical Research Organization, Ouagadougou, Burkina Faso; ${ }^{8}$ Centre Hospitalier Universitaire de Tengandogo, Ouagadougou, Burkina Faso

*These authors contributed equally to this work

Correspondence: Toussaint Rouamba Institut de Recherche en Sciences de la Santé (CNRST-IRSS), 528, Avenue Kumda-Yoore, BP 218 Ouagadougou CMS II, Ouagadougou,

Nanoro, Burkina Faso

Tel +22666653204

Email rouambatoussaint@gmail.com
Introduction: Though chloroquine derivatives are used in the treatment of coronavirus disease 2019 (COVID-19) in many countries worldwide, doubts remain about the safety and efficacy of these drugs, especially in African communities where published data are scarce. Methods: We conducted an observational prospective cohort study from April 24 to September 03, 2020, in Burkina Faso to assess (as primary outcome) the clinical, biological, and cardiac (electrocardiographic) safety of chloroquine or hydroxychloroquine plus azithromycin administered to COVID-19 patients. The main secondary outcomes were all-cause mortality and median time of viral clearance.

Results: A total of 153 patients were enrolled and followed for 21 days. Among patients who took at least one dose of chloroquine or hydroxychloroquine (90.1\% [138/153]), few clinical adverse events were reported and were mainly rash/pruritus, diarrhea, chest pain, and palpitations. No statistically significant increase in hepatic, renal, and hematological parameters or electrolyte disorders were reported. However, there was a significant increase in the QTc value without exceeding $500 \mathrm{~ms}$, especially in those who received chloroquine phosphate. Three adverse events of special interest classified as serious (known from chloroquine derivatives) were recorded namely pruritus, paresthesia, and drowsiness. One case of death occurred. The average onset of SARS-CoV-2 PCR negativity was estimated at 7.0 (95\% CI: 5.0-10.0) days. Conclusion: Hydroxychloroquine appeared to be well tolerated in treated COVID-19 patients in Burkina Faso. In the absence of a robust methodological approach that could generate a high level of scientific evidence, our results could at least contribute to guide health decisions that should be made based on different sources of scientific evidence including those from our study.

Keywords: SARS-CoV-2, chloroquine, hydroxychloroquine, azithromycin, safety, virus shedding

\section{Introduction}

Like other sub-Saharan African (SSA) countries, which are characterized by a relatively poorly operating health system, Burkina Faso is facing the consequences of the coronavirus disease 2019 (COVID-19) pandemic since March 9, 2020. ${ }^{1,2}$ Without any scientifically validated vaccines and drugs during the first year of the outbreak, control strategies were essentially based on physical barrier measures through the isolation of infected cases (suspected or confirmed) and the lockdown of the populations. ${ }^{3}$ 
Nevertheless, it was reported that existing approved drugs such as remdesivir and hydroxychloroquine $(\mathrm{HCQ})^{4,5}$ had shown in vitro activity against bacterial, fungal, and viral pathogens, including SARS-CoV-2, the causal agent of COVID-19. ${ }^{6,7}$ As a result, studies were carried out to assess the efficacy and safety of antiviral drugs $^{8-13}$ and hydroxychloroquine alone or in combination with other drugs to potentiate its therapeutic effect. The first published results seemed to support a positive effect of hydroxychloroquine in the treatment of COVID-19. ${ }^{14-17}$ These findings have prompted its adoption as COVID-19 therapy in many countries throughout the world, based on this scientific presumption.

However, the scientific community was divided on the efficacy and safety of HCQ in the treatment of COVID-19 patients. Indeed, when most of the retrospective studies claimed an improvement in clinical conditions in patients treated with HCQ, ${ }^{14-16,18-23}$ randomized controlled studies were discontinued ${ }^{24-28}$ because of their apparent ineffectiveness with a tendency to increase overall mortality. Discrepancies of findings between observational and randomized controlled studies may be explained by differences in the design and/or methodological approach with an impact on the level of the scientific evidence. ${ }^{29}$

To reinforce the existing control measures which aim at interrupting the transmission cycle of SARS-CoV-2, Burkina Faso has officially adopted a COVID-19 treatment policy with a systematic drug treatment regimen combining chloroquine (CQ) or HCQ with azithromycin (AZ). This treatment regimen has been adopted by Burkina Faso Ministry of Health based on the fact that data on dosage, drug interactions, and profile of CQ and AZ, including the adverse effects, were sufficiently known and available, particularly in the African population. ${ }^{4,17}$

The purpose of our study named CHLORAZ was to monitor and assess the adopted treatment regimens in order to generate some findings and recommendations based on scientific presumptions. Specifically, the study aimed to evaluate the clinical, biological, and cardiac (electrocardiographic) safety among patients treated with HCQ or CQ combined with $\mathrm{AZ}$, and to estimate the median time of viral clearance and assess all-cause mortality outcomes.

\section{Materials and Methods}

\section{Study Sites, Design, and Participants}

The study was carried out from April 24, 2020 to September 03, 2020, by a multidisciplinary "COVID-19-
BF research" team composed of researchers (Institut de Recherche en Sciences de la Santé and Centre Muraz) and university hospital clinicians from CHU-Tengandogo in Ouagadougou and CHU- Sourou Sanon in BoboDioulasso. The study was conducted in the two main cities of Burkina Faso, Ouagadougou and Bobo-Dioulasso, which are the two main epicenters of the outbreak.

As the study design was a prospective observational cohort study, the research team did not administer any treatment to patients. In other words, the treatments were administered by the clinical staff dedicated to the management of COVID-19 patients in the participating hospitals.

The study included all patients diagnosed by PCR as SARS-CoV-2 positive at national influenza reference laboratory (NIRL) by the participating hospitals clinical management team. Written informed consent was obtained from participants prior to data collection start.

\section{Study Procedures}

After enrolment, patients were followed-up for 21 days for clinical adverse events (AEs) as well as electrocardiographic (ECG) and biological parameters assessment (Table 1).

All patients enrolled were assessed during the first 10 days and then at day 14 and 21 at the hospital level (Table 1). At each visit, medical history since the last visit (including treatments taken), current signs and symptoms, if any, were collected. In addition, a nasopharyngeal swab was taken on days $0,3,7,10,14$, and 21 to assess the trend of the viral clearance. When the SARS-CoV-2 test was still positive on day 21 , additional samples were taken (once a week) until the test became negative, in accordance with the national standard protocol for the management of COVID-19 patients in Burkina Faso.

An ECG via 12-lead was performed on day 0, before the administration of the treatment, and was repeated on days 7 and 14. For patients with abnormal results, additional ECGs were performed weekly until normalization, or the patient was recommended to contact a physician or cardiologist to confirm the normalization of the ECG after the end of the follow-up.

Laboratory tests, namely, hematology (hemoglobin, red blood cell count, total white blood cell count, platelet count) and biochemistry (total bilirubin, aspartate transaminase [AST] and alanine transaminase [ALT], creatinine, potassium, calcium) were also measured on days 0,7 , and 14.

Regarding the study drug administration, as recommended in the Ministry of Health's treatment guidelines, 
the HCQ was given at $200 \mathrm{mg}$, three times per day for 10 days whereas CQ was given at $250 \mathrm{mg}$, twice per day for 10 days. Regarding the azithromycin, it was administered at a dose of $500 \mathrm{mg}$ on Day 0 and $250 \mathrm{mg}$ once a day from Day 1 to Day 4, ie, 5 days of treatment in total. It is important to stress that due to the observational nature of the study, the investigators were not involved in the therapeutic management of the patients. This means that they were not involved in prescribing, administering or changing the treatments.

\section{Severity and Causality Assessment of Adverse Events}

In this study, an Adverse Event (AE) was defined in accordance with international conference on harmonization good clinical practice (ICH-GCP) standards as any untoward medical occurrence in a patient or clinical investigation subject administered a pharmaceutical product and which does not necessarily have a causal relationship with this treatment.

An assessment of severity, from the WHO Toxicity Grading Scale for Determining the Severity of Adverse Events and the Division of Microbiology and Infectious Diseases of the National Institutes of Health, was used to evaluate the severity (intensity) of all adverse events (as in mild, moderate, severe or potentially life-threatening). ${ }^{30}$

The causality assessment, ie, the relationship between the study drug and AEs, regardless of their severity, was assessed through the World Health Organisation UPPSALA MONITORING CENTRE (WHO-UMC) system for standardised case causality assessment. ${ }^{31}$ Practically, the causality assessment was performed by trained physicians, based mainly on clinical judgment, possible alternative causes (eg, concomitant therapies, concomitant diseases, etc.), time of occurrence relative to the treatment and available information on the study drug. According to the WHO-UMC, the AEs were classified into six categories: Certain, Probable/Likely, Possible, Unlikely, Conditional/Unclassified, and Unassessable/Unclassifiable.

\section{Outcome Measures}

The primary outcomes, assessed up to 21 days after the patient enrolment, were the clinical, biological, and ECG safety of HCQ or CQ + AZ combination treatment in standard conditions of use, assessed through the incidence of AEs. Outcomes included also median time to SARSCoV-2 test negativity, the proportion of individuals who cleared the virus (defined as one negative swab since baseline) at days 3, 7, 14, 21, and death from any cause.

All adverse events, both serious and non-serious, were reported as per local regulatory requirements. These adverse events (AEs) included significant changes in vital signs, biochemical and hematological parameters, and electrocardiograms (ECGs). When the corrected QT interval by Fredericia (QTcF) was greater than 500 milliseconds or increased by 60 milliseconds from the baseline reading, the study medication was withheld, and the ECG

Table I Study Scheduled Visits

\begin{tabular}{|c|c|c|c|c|c|c|c|c|c|c|c|c|}
\hline \multirow[t]{2}{*}{ Follow-Up Parameters } & \multicolumn{12}{|c|}{ Rhythm (Day) } \\
\hline & 0 & $\mathbf{I}$ & 2 & 3 & 4 & 5 & 6 & 7 & 8-9 & 10 & 14 & 21 \\
\hline Informed consent & $x$ & & & & & & & & & & & \\
\hline History (symptoms) & $x$ & & & $x$ & & & & $x$ & & $x$ & $x$ & \\
\hline Examination (clinical) & $x$ & & & $x$ & & & & $x$ & & $x$ & $x$ & \\
\hline Vital signs & $x$ & & & $x$ & & & & $x$ & & $x$ & $x$ & \\
\hline Blood pressure & $x$ & & & $x$ & & & & $x$ & & $x$ & $x$ & \\
\hline Body temperature & $x$ & & & $x$ & & & & $x$ & & $x$ & $x$ & \\
\hline Electrocardiogram (ECG) & $x$ & & & & & & & $x$ & & & $x$ & \\
\hline SARS-CoV-2 rRT-PCR & $x$ & & & $x$ & & & & $x$ & & $x$ & $x$ & $x^{*}$ \\
\hline $\mathrm{CQ}$ or $\mathrm{HCQ}$ treatment & $x$ & $x$ & $x$ & $x$ & $x$ & $x$ & $x$ & $x$ & $x$ & $x$ & & \\
\hline Azithromycin treatment & $x$ & $x$ & $x$ & $x$ & $x$ & & & & & & & \\
\hline Adverse drug reactions & $x$ & & & $x$ & & & & $x$ & & $x$ & $x$ & $x$ \\
\hline Concomitant medications & $x$ & & & $x$ & & & & $x$ & & $x$ & $x$ & \\
\hline Hematology & $x$ & & & & & & & $x$ & & & $x$ & \\
\hline Biochemistry & $x$ & & & & & & & $x$ & & & $x$ & \\
\hline
\end{tabular}

Note: *If the test was positive on Day 2I, the test was repeated until it became negative.

Abbreviation: rRT-PCR, real time reverse transcriptase polymerase chain reaction. 
repeated. Additional outcomes of interest consisted of adverse events of special interest (AESI) related to cardiotoxicity, neurotoxicity, nephrotoxicity, hepatotoxicity, skin reactions or phototoxicity.

\section{Data Entry and Statistical Analysis}

Data were collected on individual paper case report forms (CRFs) from the source documents (patient medical records) by study physicians and were double-entered in an electronic database developed on Open Clinica ${ }^{\circledR}$. The final database for the analysis was obtained after the resolution of all queries raised by the data manager.

Categorical variables were represented as frequencies and percentages, continuous variables as means with standard deviations, or median and interquartile ranges (IQRs), as well as minimum and maximum values where appropriate.

Data showing the change of the biological parameters and the QTcF values were presented graphically. For each parameter, three graphs were plotted: i) a plot of the change of the individual values observed for each patient at the different visits, ii) boxplots showing the change of the distribution of the median values of the parameters at the different visits, iii) a plot of the change of the mean (and the 95\% confidence interval) of the parameters at the different visits.

Clinical adverse events were presented according to their type, frequency, severity, and potential relationship to the study drug. For the evaluation of the change in biological and ECG parameters during the follow-up, we performed comparison tests for repeated measures (at days 0, 7, and 14). Thus, the non-parametric Friedman test was used, and a p-value less than 0.05 was considered as significant.

For the analysis of the viral clearance, the KaplanMeier survival curve was constructed to estimate the probability of recovery (negative rRT-PCR result) and estimate the median time to have a negative PCR result (viral clearance).

\section{Ethical Aspects}

Ethical approval of the protocol was obtained from the Burkina Faso Health Research Ethics Committee (Deliberation No. 2020-000101/MS/MESRSI/CERS). The purpose and procedures of the study were explained to potential participants by study staff. Participants who met the inclusion criteria and agreed to participate in the survey were asked to provide written informed consent.
This study was conducted in accordance with the Declaration of Helsinki.

This study is registered with ClinicalTrials.gov: NCT04445441.

\section{Results}

\section{Patient Characteristics at Inclusion}

A total of 153 patients with COVID-19 were included between April 24 and September 03, 2020 (Table 2). Of these, $75 \%$ were under 49 years of age, and the mean age was 36.1 years. Two out of three patients were males $(66.0 \%)$, and $45.9 \%$ of patients had a university-level as education. Ten patients received $\mathrm{CQ}+\mathrm{AZ}$, whereas 128 patients received $\mathrm{HCQ}+\mathrm{AZ}$. A total of 14 participants did not take the chloroquine-based treatment for various reasons, mainly due to known allergies or refusal.

\section{Clinical Signs and Symptoms at Inclusion}

The distribution of vital signs at inclusion according to treatment is shown in Table 3. The median oxygen saturation $(\mathrm{SaO} 2)$ was $98.0 \%$, which indicates that the patients did not have an obvious major respiratory distress. The minimum oxygen saturation in the included patients was $90 \%$. About $13.2 \%$ of the patients had a temperature of $37.5^{\circ} \mathrm{C}$ or higher at inclusion.

Furthermore, at inclusion, influenza-like symptoms were present, namely cough $(14.4 \%)$, fever $(13.2 \%)$, headache $(10.5 \%)$, runny nose $(6.5 \%)$, sore throat $(5.9 \%)$, and fatigue $(3.9 \%)$. In addition, shortness of breath, anosmia, and ageusia were found in $6.5 \%(10 / 153), 5.2 \%(8 / 153)$, and $3.9 \%(6 / 153)$ of patients, respectively.

Besides the clinical signs and symptoms, regarding the co-medication use by the study participants during the follow-up period, the antihypertensives were the most commonly used drug, followed by vitamin $\mathrm{C}$, and antidiabetics. The distribution of co-medications is shown in Table S1.

\section{Clinical Tolerance: Clinical Adverse Events}

Clinical tolerance was assessed in all patients who received at least one dose of $\mathrm{CQ} / \mathrm{HCQ}+\mathrm{AZ}$. The distribution of the occurrence of adverse events according to chloroquine-based treatment is presented in Table 4. A few clinical AEs were reported during participants' follow-up. Thus, a total of 42 AEs were reported among 23 participants. Of these, 97.6\% (41/42) of AEs occurred 
Table 2 Characteristics of the Population at Inclusion

\begin{tabular}{|c|c|c|c|c|}
\hline \multirow[t]{2}{*}{ Characteristics } & \multirow[t]{2}{*}{ Total No. $=153$} & \multicolumn{3}{|c|}{ Treatment } \\
\hline & & HCQ + AZ No. $=128$ & $C Q+A Z$ No. $=10$ & Not Treated No. $=14$ \\
\hline \multicolumn{5}{|l|}{ Age } \\
\hline Median (IQR) & $33.0(16.2)$ & $32.0(16.0)$ & $27.5(13.8)$ & $42.0(11.0)$ \\
\hline Min - max & $16.0-89.0$ & $16.0-89.0$ & $21.0-48.0$ & $22.0-61.0$ \\
\hline \multicolumn{5}{|l|}{ Sex, n(\%) } \\
\hline Female & $52(34.0)$ & $4 \mid(3 \mid .8)$ & $2(20.0)$ & $9(64.3)$ \\
\hline Male & $101(66.0)$ & $88(68.2)$ & $8(80.0)$ & $5(35.7)$ \\
\hline \multicolumn{5}{|l|}{ Marital status, n(\%) } \\
\hline Single & 7I (5I.I) & 61 (52.6) & $6(60.0)$ & $4(30.8)$ \\
\hline In couple & $68(48.9)$ & $55(47.4)$ & $4(40.0)$ & $9(69.2)$ \\
\hline \multicolumn{5}{|l|}{ Profession, $n(\%)$} \\
\hline Informal sector & 17 (II.7) & $16(13.0)$ & $0(0.0)$ & I (8.3) \\
\hline Teacher & $6(4.1)$ & $5(4.1)$ & $I(10.0)$ & $0(0.0)$ \\
\hline Health worker & $15(10.3)$ & $12(9.8)$ & $0(0.0)$ & $3(25.0)$ \\
\hline Farmer & $5(3.4)$ & $4(3.3)$ & $0(0.0)$ & $\mathrm{I}(8.3)$ \\
\hline Housewife & $15(10.3)$ & 14 (I I.4) & $0(0.0)$ & I (8.3) \\
\hline Student & $34(23.4)$ & $30(24.4)$ & $4(40.0)$ & $0(0.0)$ \\
\hline Retired & $3(2.1)$ & $2(1.6)$ & $0(0.0)$ & I (8.3) \\
\hline Other & $50(34.5)$ & $40(32.5)$ & $5(50.0)$ & $5(4 I .7)$ \\
\hline \multicolumn{5}{|l|}{ Educational level, n(\%) } \\
\hline Non-formal education & $3(2.1)$ & $2(1.6)$ & $0(0.0)$ & I (7.7) \\
\hline No education at all & $18(12.3)$ & 17 (13.8) & $0(0.0)$ & I (7.7) \\
\hline Primary education & $12(8.2)$ & II (8.9) & $0(0.0)$ & I (7.7) \\
\hline Post-primary education & $45(30.8)$ & $37(30.1)$ & $4(40.0)$ & $4(30.8)$ \\
\hline University & $67(45.9)$ & $55(44.7)$ & $6(60.0)$ & $6(46.2)$ \\
\hline Weight (kg), Mean (SD) & $72.8(15.2)$ & $73.2(15.4)$ & $64.3(15.6)$ & $75.8(12.4)$ \\
\hline Height (cm), Median (IQR) & $170.0(9.0)$ & $171.0(9.0)$ & $167.0(9.0)$ & I $70.5(5.5)$ \\
\hline Body mass index $\left(\mathbf{k g} / \mathrm{m}^{2}\right)$ Mean (SD) & $25.6(6.4)$ & $25.8(6.8)$ & $22.3(4.3)$ & $26.4(4.1)$ \\
\hline \multicolumn{5}{|l|}{ Charlson co -morbidity index, $n(\%)$} \\
\hline 0 & $126(83.0)$ & $103(80.6)$ & $10(100.0)$ & $13(92.9)$ \\
\hline $1-2$ & $22(14.4)$ & $21(16.3)$ & $0(0.0)$ & I (7.I) \\
\hline $3-4$ & $4(2.6)$ & $4(3.1)$ & $0(0.0)$ & $0(0.0)$ \\
\hline
\end{tabular}

in 22 participants who received chloroquine. Among participants who received chloroquine, AEs were mainly skin disorders (rash and pruritus). Other AEs such as diarrhea, chest pain, dysarthria, and palpitations were reported. Among patients who received HCQ, the events were mainly skin disorders (pruritus). Other AEs such as diarrhea, chest pain, anemia, headache, jaundice (hyperbilirubinemia), pleurisy, pneumonitis, and tachycardia were also reported. It was noticeable that a few AEs were also reported among patients who did not receive any chloroquine-based treatment. These included one case of hypertension. As per WHO-UMC causality assessment criteria, two AEs (Pruritus and Diarrhea) were classified as "certain", eight (one case of chest pain, one case of skin rash, two cases of palpitations, and four cases of pruritus) as "probable", five as "possible", 15 as "unlikely", and 11 as "unrelated". The distribution of AEs according to their relationship to the study drug is presented in Table S2.

AEs with an unlikely relationship to CQ/HCQ were most common and represented 39.5\% (17/43) of all adverse events. In terms of severity, the study physicians classified 
Table 3 Distribution of Vital Signs at Inclusion by Treatment

\begin{tabular}{|c|c|c|c|c|}
\hline \multirow[t]{2}{*}{ Vital Signs } & \multirow[t]{2}{*}{ Total } & \multicolumn{3}{|c|}{ Treatment } \\
\hline & & $\mathrm{HCQ}+\mathrm{AZ}$ & $\mathbf{C Q}+\mathbf{A Z}$ & Not Treated \\
\hline Oxygen saturation (SaO2), Median (IQR) & $98.0(3.0)$ & $98.0(0.0)$ & $97.5(2.0)$ & $99.0(1.0)$ \\
\hline Temperature $\left({ }^{\circ} \mathrm{C}\right)$, Median (IQR) & $36.9(0.8)$ & $37.2(0.7)$ & $36.9(0.8)$ & $37.2(0.4)$ \\
\hline \multicolumn{5}{|l|}{ Fever (Temperature $\left.\geq 37.5^{\circ} \mathrm{C}\right), \mathrm{n}(\%)$} \\
\hline Yes & $20(13.2)$ & $4(40.0)$ & $13(10.2)$ & $3(2 \mid .4)$ \\
\hline No & I 32 (86.8) & $6(60.0)$ & $115(89.8)$ & II (78.6) \\
\hline Respiratory rate (c/min), Median (IQR) & $19.0(3.5)$ & $21.0(3.5)$ & $19.0(2.5)$ & $18.5(2.2)$ \\
\hline Pulse (pulsations/min), Mean (SD) & $84.8(15.1)$ & $96.2(14.5)$ & $84.6(14.7)$ & $78.6(14.8)$ \\
\hline \multicolumn{5}{|l|}{ Blood pressure $(\mathrm{mmHg})$, Median (IQR) } \\
\hline Systolic & $126.5(20.8)$ & $110.0(18.0)$ & $129.0(21.0)$ & I27.5 (I7.8) \\
\hline Diastolic & $81.0(17.0)$ & $75.0(10.0)$ & $82.0(17.0)$ & $86.5(19.5)$ \\
\hline
\end{tabular}

the majority of AEs as non-severe. Three SAEs of special interest $[2.1 \%(3 / 139)]$, known to be related to chloroquine derivatives, were recorded. These included one case of pruritus-type skin reaction, one case of neurotoxicity (cramp/ paresthesia), and one case of drowsiness. The outcomes of these events resulted in complete recovery without sequelae.

\section{Biological Tolerance}

During the 21-days follow-up period, the investigation of laboratory tests at days 0,7 , and 14 did not report any statistically significant change in liver function (AST, total bilirubin) and kidney function (creatinine). However, there was a statistically significant decrease in the median value of ALT and blood glucose, although they were not under the lower limit of the normal value range.

Regarding the electrolytes balance, there was a nonsignificant increase of the potassium median value (from day 0 to day 14) but did not reach the upper limit of the normal value range. However, the analysis of the change of potassium median values between day 0 and day 7 showed a significant increase without reaching the upper limit of the normal value range.

No increase in calcium median values was noted during the follow-up (from day 0 to day 14) among the patients included in the cohort.

The change of biochemical parameters between day 0 and day 14 is presented in Figures S1-S7.

Regarding the change of hematological parameters, there was a non-significant decrease in the median value of hemoglobin from day 0 to day 7 . However, on day 14, there was a tendency to return to the normal value range (Figure S8). Furthermore, there was no significant change in white blood cells (Figure S9) and platelet values . Regarding the change in platelet values, there was an increase in the median value of platelets during the followup, without exceeding the upper limit of the normal range values (Figure S10).

\section{Cardiac Tolerance}

At inclusion, three patients (two HCQ-treated and one untreated) had QTcF values $>500 \mathrm{~ms}$, but these values were normalized by day 3 of follow-up for two patients. The QTcF of the third patient decreased on day 7 but was still above $500 \mathrm{~ms}$. Among patients who had a normal QTcF value at baseline, an increase in QTcF values ( $>500 \mathrm{~ms}$ ) was observed in one patient at day 3 , resulting in the discontinuation of the treatment. The median QTcF value was significantly increased overall in patients treated with CQ but did not exceed 500ms (Figure 1).

A total of seven treated patients and one untreated patient exhibited an increase of at least $60 \mathrm{~ms}$ in QTcF from baseline. In addition, after investigation of patients with clinical signs suggestive of cardiotoxicity, one patient with chest pain had an increase in QTcF from baseline $>60 \mathrm{~ms}$.

\section{Viral Clearance}

The median time to SARS-CoV-2 negativity after the enrolment of patients in the study was estimated at 7.0 days (95\% CI: 5.0-10.0). Before day 7, specifically at day 3 after the enrolment, $63.3 \%(95 \% \mathrm{CI}: 54.3-75.1)$ of 
Table 4 Adverse Events Reported During Participant's Follow-Up According to Treatment Received

\begin{tabular}{|c|c|c|c|c|c|}
\hline \multirow[t]{2}{*}{ Adverse Events, n (\%) } & \multirow[t]{2}{*}{ Total } & \multicolumn{3}{|c|}{ Treatment } & \multirow[t]{2}{*}{ p value } \\
\hline & & HCQ + AZ & $C Q+A Z$ & Not Treated & \\
\hline \# of adverse events & 42 & 34 & 7 & 2 & 0.71 \\
\hline \multicolumn{6}{|l|}{ Name of adverse events } \\
\hline Pruritus & 5 & $3(60.0)$ & $2(40.0)$ & $0(0.0)$ & \\
\hline Diarrhea & 4 & $3(75.0)$ & I (25.0) & $0(0.0)$ & \\
\hline High blood pressure & 4 & $3(75.0)$ & $0(0.0)$ & I (25.0) & \\
\hline Headache & 3 & $3(100)$ & $0(0.0)$ & $0(0.0)$ & \\
\hline Chest pain & 3 & $2(66.7)$ & I (33.3) & $0(0.0)$ & \\
\hline Jaundice & 2 & $2(100)$ & $0(0.0)$ & $0(0.0)$ & \\
\hline Palpitations & 2 & I (50.0) & I (50.0) & $0(0.0)$ & \\
\hline Anemia & I & $I(100)$ & $0(0.0)$ & $0(0.0)$ & \\
\hline Anorexia & 1 & $I(100)$ & $0(0.0)$ & $0(0.0)$ & \\
\hline Cramp + paresthesia & 1 & $I(100)$ & $0(0.0)$ & $0(0.0)$ & \\
\hline Diarrhea + abdominal pain & 1 & I (100) & $0(0.0)$ & $0(0.0)$ & \\
\hline Breathing difficulties & 1 & $I(100)$ & $0(0.0)$ & $0(0.0)$ & \\
\hline Dysarthria & 1 & $0(0.0)$ & $I(100)$ & $0(0.0)$ & \\
\hline Epigastralgia & 1 & $I(100)$ & $0(0.0)$ & $0(0.0)$ & \\
\hline Skin rash & 1 & $0(0.0)$ & $I(100)$ & $0(0.0)$ & \\
\hline Insomnia & 1 & $I(100)$ & $0(0.0)$ & $0(0.0)$ & \\
\hline Renal failure & 1 & $I(100)$ & $0(0.0)$ & $0(0.0)$ & \\
\hline Septic monoarthritis & 1 & $I(100)$ & $0(0.0)$ & $0(0.0)$ & \\
\hline Uncomplicated malaria & 1 & $I(100)$ & $0(0.0)$ & $0(0.0)$ & \\
\hline Pleurisy & 1 & $I(100)$ & $0(0.0)$ & $0(0.0)$ & \\
\hline Pneumonia & 1 & $I(100)$ & $0(0.0)$ & $0(0.0)$ & \\
\hline Somnolence & 1 & $I(100)$ & $0(0.0)$ & $0(0.0)$ & \\
\hline Infectious syndrome & 1 & $I(100)$ & $0(0.0)$ & $0(0.0)$ & \\
\hline Tachycardia & 1 & $I(100)$ & $0(0.0)$ & $0(0.0)$ & \\
\hline Cough & 1 & $I(100)$ & $0(0.0)$ & $0(0.0)$ & \\
\hline Vomiting & 1 & I (100) & $0(0.0)$ & $0(0.0)$ & \\
\hline
\end{tabular}

patients were positive. At day 14, 24.1\% (95\% CI 16.235.8 ) of patients were still positive, and $16.6 \%$ (95\% CI: 9.9-27.8) at day 21. Thus, at day 30 and 37, 5.6\% (95\% CI: $2.1-16.3)$ and $2.9 \%$ (95\% CI: $0.5-16.6)$ of patients were still positive to SARS-CoV-2, respectively. The Kaplan Meier curve of viral clearance is presented in Figure 2, below.

\section{Death from Any Cause}

During the follow-up, one death occurred. This was a 34year-old female patient with a known medical history of chronic kidney disease, congestive hypertrophic heart disease, and arterial hypertension treated with furosemide, amlodipine, and atenolol. She was admitted to the hospital with an altered general condition associated with respiratory distress, chest pain with regular sinus rhythm, anemia, and an infectious syndrome.

\section{Discussion}

In clinical research, it is clearly demonstrated that randomized clinical trials are the best way to establish the safety/ efficacy of a treatment. ${ }^{29}$ However, given the health emergency context where it was difficult to set up such type of study (partly due to the national policy recommendation of administering systematically the $\mathrm{HCQ} / \mathrm{CQ}+\mathrm{AZ}$ combination treatment to all COVID-19 patients), this prospective observational study was set up to provide presumptive scientific information on i) the tolerance profile of HCQ/ CQ treatment and, ii) the median time of viral clearance among patients with COVID-19 in Burkina Faso. 

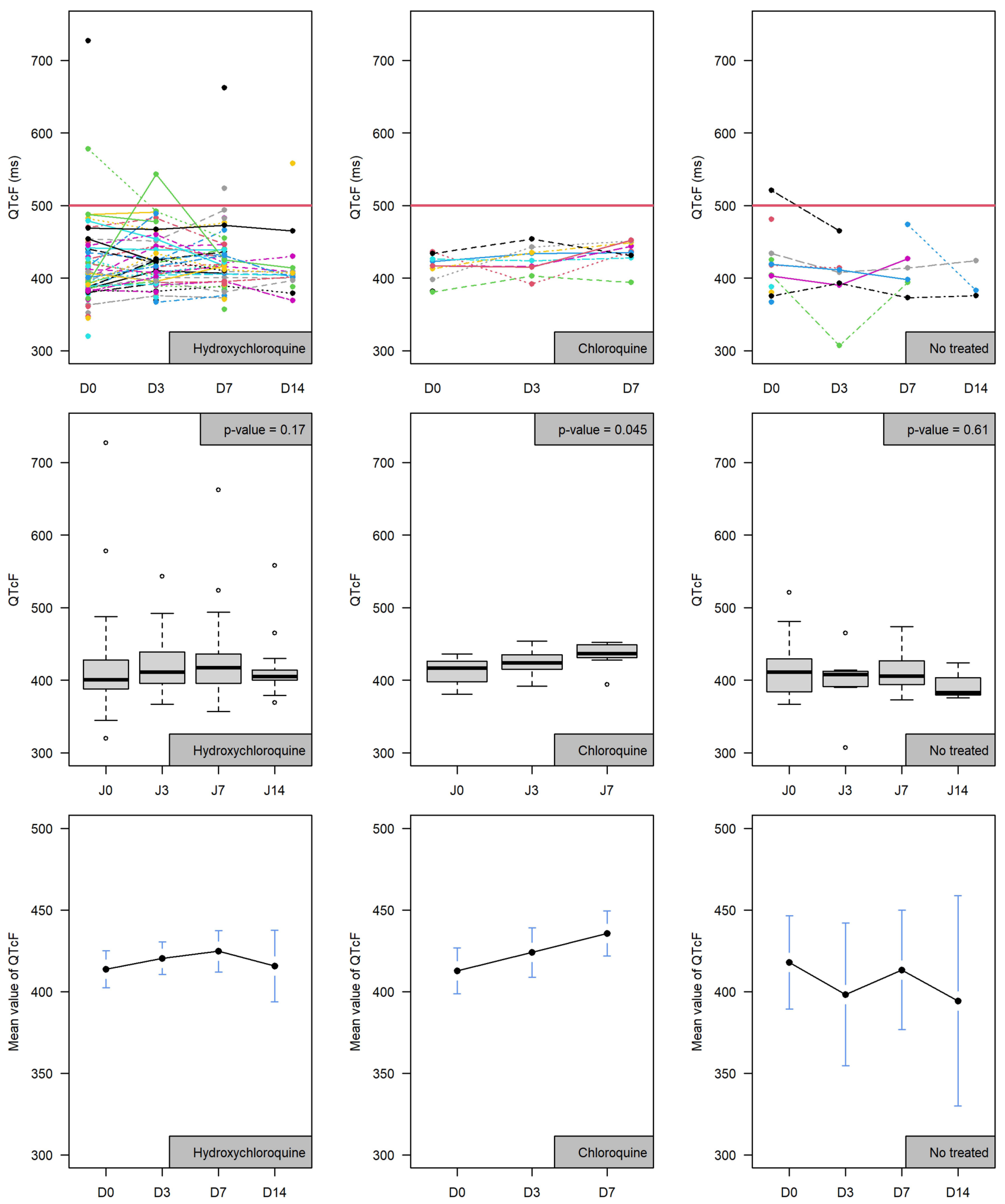

Figure I Change in corrected QT interval by Fredericia (QTcF) values during follow-up according to the treatment received 


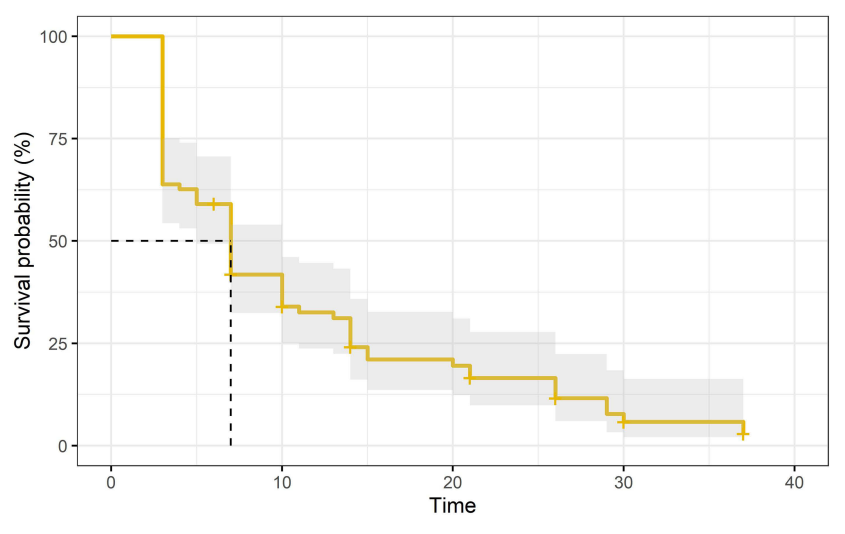

Figure 2 Viral cleareance during the follow-up

\section{Tolerance Profile of HCQ/CQ Treatment} Our findings showed that the combination of $\mathrm{CQ} / \mathrm{HCQ}+$ AZ was relatively well tolerated clinically, although some adverse events well known from chloroquine derivatives (pruritus, cramp/paresthesia, and sleep disorders) were reported with more or less discomforting effects. In addition, regarding biological tolerance, the results showed a significant increase in the median potassium value between day 0 and day 7 (Friedman rank-sum test, $\mathrm{p}=$ 0.01 ) without exceeding the upper limit of the normal value range. We did not observe any clinically significant change in the other biochemical parameters and the trend was similar for the hematological parameters. There was a significant increase in the QTc value compared with the baseline values without exceeding $500 \mathrm{~ms}$, especially in those who received chloroquine phosphate. These results (in particular, increased cardiac risks, ie, QTc prolonged), are consistent with other studies conducted in many countries. $^{12,32-35}$

According to the literature, both hydroxychloroquine and chloroquine were associated with QT interval prolongation, increased risk of "torsade de pointes", ventricular arrhythmias, and sudden cardiac death. ${ }^{13,36-38}$ Azithromycin was also shown to prolong the QT interval in patients, most notably among older people. ${ }^{39-42}$ With regard to cardiac safety assessed in this study (specifically QTcF interval), we observed in one patient a QTcF interval value $>500 \mathrm{~ms}$ on day 3 after treatment. This QT interval prolongation occurred in a 42-year-old patient with two comorbidities (chronic kidney disease associated with hypertension) and treated with amlodipine. The prolongation of the QTcF interval in patients with COVID-19 treated with chloroquine-based molecules (in combination with or without a second-generation macrolide) has been widely reported in several studies (observational and randomized clinical trial). ${ }^{13,38}$ Although chloroquine or hydroxychloroquine are generally safe when used for approved indications such as autoimmune diseases or malaria, the safety and benefits of these regimens in the management of COVID-19 were rarely reported in the African context. While most retrospective studies conducted outside the African continent have reported clinical improvement with HCQ, randomized controlled trials have been discontinued ${ }^{24-28}$ due to a trend towards increased overall mortality. In the context of $\mathrm{CQ} / \mathrm{HCQ}$ use in the treatment of SARS-CoV-2 infection, several studies have attempted to provide an explanation for QTc prolongation. Firstly, SARS-CoV-2 infection may itself attack cardiac myocytes, leading to cardiac disorders such as arrhythmias. Indeed, early studies suggested that COVID-19 was associated with a high incidence of cardiac arrhythmias. ${ }^{43}$ Secondly, electrolyte disturbances such as hyperkalemia (as observed in this study), or acute kidney injury, frequently occur during COVID-19. ${ }^{44}$ These abnormalities may directly increase the risk of cardiac arrhythmia by potentiating the QTc prolongation induced by HCQ. Thirdly, the cumulative effect of an additional drug with the potential to prolong the QTc interval may be another explanation (HCQ + azithromycin combination). ${ }^{45}$

\section{Viral Clearance Among Patients with COVID-19 in Burkina Faso}

Our study showed that the median time of viral clearance was 7 days among the enrolled patients. This time seemed relatively short compared to the median time of viral clearance reported in a systematic review and metaanalysis that estimated this time of clearance of SARSCoV-2 RNA to be 17.0 days. ${ }^{46} \mathrm{~A}$ study carried-out in Ethiopia found that the median duration of viral clearance from each participant's body was 19 days, but the range was wide: 2 to 71 days. ${ }^{47}$ However, our findings are similar to the results reported in other observational studies. Indeed, the median duration of SARS-CoV-2 negativity was estimated to be 6 days (IQR: 04-10) in a study conducted by Chen et al in China. ${ }^{48}$ Similar median durations of viral clearance were also reported by $\mathrm{Hu}$ et al (06 days; IIQ: 02-12) ${ }^{49}$ Sakurai et al (09 days; IQR: 06-11), ${ }^{50}$ and Yang et al (08 days; IQR: 03-12). ${ }^{51}$ In our study, in the absence of a control group, it was difficult to attribute this shortened time of negativity to chloroquine treatment only. Several factors could also explain this median time 
of seven days, including the young age of our study population and the high percentage of "paucisymptomatic" cases. Indeed, studies have shown that viral clearance seems to be shorter in young people compared to the elderly and also shorter in asymptomatic people compared to symptomatic patients. ${ }^{4,51-53}$ In our setting, several days certainly elapse between the onset of disease and the date of diagnosis for many patients, whereas the viral load of SARS-CoV-2 in the upper respiratory tract appears to peak during the first week of infection, and no studies have found live virus beyond day 9, although viral loads are still high. ${ }^{46}$ Furthermore, it is important to stress that the duration of viral clearance may depend greatly on the SARS-CoV-2 variant. Therefore, we have no robust arguments that can explain the short duration of viral clearance in our observational study.

Our study has several limitations, which are mainly related to its design. First, the causality assessment tools of AEs could produce some bias (relationship likelihood or disagreements between assessors). It is noticeable that for AEs causality assessment, there are several structured tools, but none has gained universal acceptance (none of these tools, were shown to produce a precise and reliable quantitative estimation of relationship likelihood). ${ }^{54}$ In our study, the WHO-UMC was used, this could limit the disagreement between assessors. ${ }^{55}$ Second, the absence of a control group did not allow us to establish scientific evidence of causal association between the above-mentioned therapeutic regimens and the reduction of the time of viral load negativity and mortality due to COVID-19. Third, in this study, the treatment was administered by the hospital's routine management team (or at home), so, the study team did not evaluate the adherence/compliance of the treatment taken by patients. This could lead to an under- or over-estimation of the frequency of adverse events. Fourth, if we consider that the source population, ie, the one to which we wish to generalize our results, is all the COVID-19 patients in the two main cities of the country, our sampling approach is the one by convenience. In fact, many patients did not visit the sites from where our data have been collected. This type of selection bias could lead to an overestimating or underestimating the situation. Our results should be interpreted with these strengths and limitations in mind.

\section{Conclusion}

This observational cohort study showed that no lifethreatening safety issues were observed with the use of $\mathrm{CQ} / \mathrm{HCQ}+\mathrm{AZ}$ combination for the treatment of COVID-
19 in Burkina Faso. Although our findings are consistent with data from several other published observational studies, they are inconsistent with data from randomized clinical trials regarding the safety of $\mathrm{CQ} / \mathrm{HCQ}+\mathrm{AZ}$ combination treatment which appears to be well tolerated in our study despite an increase in the QTc interval. Close monitoring should be performed, particularly in patients with known underlying illnesses, especially those with kidney or cardiac diseases. In the absence of an evidence-based approach, our results could contribute to guide health decisions by confronting them with other existing sources. Therefore, the decision of whether or not the use of these combination treatments should be discontinued in Burkina Faso should be based on the contextualized findings presented here but also on the appropriate international recommendations that guided its introduction in the country.

\section{Data Sharing Statement}

Anonymised participant data could be made available, upon requests directed to the corresponding author. If agreed, data can be shared through a secure online platform after signing a data transfer agreement.

\section{Acknowledgments}

We thank all the participants in the study and the staff of two participating University hospitals (Tengandogo and Sourou Sanon). The study was funded by the "Fond National de la Recherche et de 1 'Innovation pour le Developpement (FONRID)" from Burkina Faso Ministry of Higher Education, Scientific Research and Innovation (MESRSI).

The Burkina Faso CHLORAZ Study Group included the Centre Hospitalier Universitaire de Tengandogo team ( $\mathrm{Pr}$ SANOU Adama, Dr Abdoul Risgou OUEDRAOGO, Dr KONATE Lassina, Dr DIALlO Issa, Dr SOME Hippolyte, Dr ROUAMBA Nadège, Dr SERE Lassina, Dr YANOGO Donald, Mr GUIGMA Nicolas, Mr KABRE Moussa and Mr BADO Gnaboe Maurice). The Clinique Princesse Sarah team (Dr MINOUNGOU Jules Christian W, Dr ZIDA Justin, Dr BARRO Djamilatou, Dr ILLA Joseph, Dr KAMBOU Bruno, Dr ILBOUDO David, Dr KI Ghislain, Dr AGBODOSSINDJI Fiacre, Dr GNAMOU Arouna, Mr KAWANE Justine, Mr KAFANDO Mathieu). The District sanitaire de Pissy team (Dr TIEMTORE Ousseni, Dr SOW Abobacar, Dr Seydou ZIDA, Mr BANHORO Honore Achille). The Equipe mobile team (Dr KABORE Mikaila, Dr NANA Harouna). The Clinical Research Unit of Nanoro (Mr HIEN S. Franck). The Centre Muraz/Institut National de Santé Publique team (ZOMA 
Aristide). Service des maladies infectieuses team (BOLY Raïnatou). Médecine Interne, Centre Hospitalier Universitaire de Sourou Sanon, Bobo-Dioulasso team (NIGNAN Issan Urbain). Service de pneumologie, Centre Hospitalier Universitaire de Sourou Sanon, Bobo-Dioulasso, Burkina Faso (OUEDRAOGO Patricia Roseline).

\section{Disclosure}

The authors report no conflicts of interest in this work.

\section{References}

1. WHO. Coronavirus Disease 2019 (COVID-19) Situation Report-51; 2020.

2. Worldometer. Coronavirus Cases. Worldometer. Available from: https://www.worldometers.info/coronavirus/coronavirus-cases /\#daily-cases. Accessed December 31, 2020.

3. Nouveau coronavirus (2019-nCoV): conseils au grand public. Available from: https://www.who.int/fr/emergencies/diseases/novelcoronavirus-2019/advice-for-public?gclid=Cj0KCQiA-

OeBBhDiARIsADyBcE5Jso8gwdIiiLhwB6Z156a4a7SgYhVf3idBz OwJIX-8j8k9yCDOwk0aAswQEALw_wcB. Accessed February 27, 2021.

4. Wang M, Cao R, Zhang L, et al. Remdesivir and chloroquine effectively inhibit the recently emerged novel coronavirus $(2019-\mathrm{nCoV})$ in vitro. Cell Res. 2020;30(3):269-271. doi:10.1038/s41422-020-0282-0

5. Rolain JM, Colson P, Raoult D. Recycling of chloroquine and its hydroxyl analogue to face bacterial, fungal and viral infections in the 21 st century. Int J Antimicrob Agents. 2007;30:297-308. doi:10.1016/ j.ijantimicag.2007.05.015

6. Drożdżal S, Rosik J, Lechowicz K, et al. FDA approved drugs with pharmacotherapeutic potential for SARS-CoV-2 (COVID-19) therapy. Drug Resist Updat. 2020;53:100719. doi:10.1016/j. drup. 2020.100719

7. Yao X, Ye F, Zhang M, et al. In vitro antiviral activity and projection of optimized dosing design of hydroxychloroquine for the treatment of Severe Acute Respiratory Syndrome Coronavirus 2 (SARS-CoV-2. Clin Infect Dis. 2020;71(15):732-739. doi:10.1093/cid/ciaa237

8. Piscoya A, Ng-Sueng LF, Parra Del Riego A, et al. Efficacy and harms of remdesivir for the treatment of COVID-19: a systematic review and meta-analysis. PLoS One. 2020;15(12):e243705. doi:10.1371/journal.pone.0243705

9. Wilt TJ, Kaka AS, MacDonald R, et al. Remdesivir for adults with COVID-19: a living systematic review for American college of physicians practice points. Ann Intern Med. 2021;174(2):209-220. doi:10.7326/M20-5752

10. Davies M, Osborne V, Lane S, et al. Remdesivir in treatment of COVID-19: a systematic benefit-risk assessment. Drug Saf. 2020;43:645-656.

11. Million M, Lagier J-C, Tissot-Dupont H, et al. Early combination therapy with hydroxychloroquine and azithromycin reduces mortality in 10,429 COVID-19 outpatients. Rev Cardiovasc Med. 2021;22 (3):1063-1072. doi:10.31083/j.rcm2203116

12. Hariyanto TI, Kristine E, Jillian Hardi C, Kurniawan A. Efficacy of lopinavir/ritonavir compared with standard care for treatment of Coronavirus Disease 2019 (COVID-19): a systematic review. Infect Disord - Drug Targets. 2021;21:e270421187364.

13. Diaz-Arocutipa C, Brañez-Condorena A, Hernandez AV. QTc prolongation in COVID -19 patients treated with hydroxychloroquine, chloroquine, azithromycin, or lopinavir/ritonavir: a systematic review and meta-analysis. Pharmacoepidemiol Drug Saf. 2021;30 (6):694-706. doi:10.1002/pds.5234
14. Gautret P, Lagier J-C, Parola P, et al. Clinical and microbiological effect of a combination of hydroxychloroquine and azithromycin in 80 COVID-19 patients with at least a six-day follow up: a pilot observational study. Travel Med Infect Dis. 2020;34:101663. doi:10.1016/j.tmaid.2020.101663

15. Lagier J-C, Million M, Gautret P, et al. Outcomes of 3737 COVID-19 patients treated with hydroxychloroquine/azithromycin and other regimens in Marseille, France: a retrospective analysis. Travel Med Infect Dis. 2020;36:101791. doi:10.1016/j.tmaid.2020.101791

16. Catteau L, Dauby N, Montourcy M, et al. Low-dose hydroxychloroquine therapy and mortality in hospitalised patients with COVID-19: a nationwide observational study of 8075 participants. Int J Antimicrob Agents. 2020;56(4):106144. doi:10.1016/j.ijantimicag.2020.106144

17. Colson P, Rolain J-M, Lagier J-C, Brouqui P, Raoult D. Chloroquine and hydroxychloroquine as available weapons to fight COVID-19. Int $J$ Antimicrob Agents. 2020;55(4):105932. doi:10.1016/j. ijantimicag.2020.105932

18. Ayerbe L, Risco-Risco C, Ayis S. The association of treatment with hydroxychloroquine and hospital mortality in COVID-19 patients. Intern Emerg Med. 2020;15:1501-1506.

19. Lammers AJJ, Brohet RM, Theunissen REP, et al. Early hydroxychloroquine but not chloroquine use reduces ICU admission in COVID-19 patients. Int $J$ Infect Dis. 2020;101:283-289. doi:10.1016/j.ijid.2020.09.1460

20. Arshad S, Kilgore P, Chaudhry ZS, et al. Treatment with hydroxychloroquine, azithromycin, and combination in patients hospitalized with COVID-19. Int J Infect Dis. 2020;97:396-403. doi:10.1016/j. ijid.2020.06.099

21. Bernaola N, Mena R, Bernaola A, et al. Observational study of the efficiency of treatments in patients hospitalized with Covid-19 in Madrid. medRxiv. 2020;2020. doi:10.1101/2020.07.17.20155960

22. Million M, Lagier J-C, Gautret P, et al. Early treatment of COVID-19 patients with hydroxychloroquine and azithromycin: a retrospective analysis of 1061 cases in Marseille, France. Travel Med Infect Dis. 2020;35:101738. doi:10.1016/j.tmaid.2020.101738

23. Yu B, Li C, Chen P, et al. Beneficial effects exerted by hydroxychloroquine in treating COVID-19 patients via protecting multiple organs. $S c i$ China Life Sci. 2021;64(2):330-333. doi:10.1007/s11427-020-1782-1

24. Reis G, Moreira Silva EADS, Medeiros Silva DC, et al. Effect of early treatment with hydroxychloroquine or lopinavir and ritonavir on risk of hospitalization among patients with COVID-19: the TOGETHER randomized clinical trial. JAMA Netw open. 2021;4 (4):e216468. doi:10.1001/jamanetworkopen.2021.6468

25. Réa-Neto Á, Bernardelli RS, Câmara BMD, et al. An open-label randomized controlled trial evaluating the efficacy of chloroquine/ hydroxychloroquine in severe COVID-19 patients. Sci Rep. 2021;11 (1):9023. doi:10.1038/s41598-021-88509-9

26. Dabbous HM, El-Sayed MH, El Assal G, et al. Safety and efficacy of favipiravir versus hydroxychloroquine in management of COVID-19: a randomised controlled trial. Sci Rep. 2021;11:1-7.

27. The RECOVERY Collaborative Group. Effect of hydroxychloroquine in hospitalized patients with Covid-19. $N$ Engl $J$ Med. 2020;383:2030-2040. doi:10.1056/NEJMoa2022926

28. Pan H, Peto R, Karim QA, et al. Repurposed antiviral drugs for COVID-19 -interim WHO SOLIDARITY trial results. medRxiv. 2020;2020. doi:10.1101/2020.10.15.20209817

29. Haute Autorité de Santé. Niveau de preuve et gradation des recommandations de bonne pratique. Etat des lieux; 2013.

30. Safety Reporting and Pharmacovigilance. NIH: National Institute of Allergy and Infectious Diseases. Available from: https://www.niaid. nih.gov/research/dmid-safety-reporting-pharmacovigilance. Accessed October 12, 2021.

31. The Uppsala Monitoring Centre. The use of the WHO-UMC system for standardised case causality assessment. Available from: https:// www.who.int/medicines/areas/quality_safety/safety_efficacy/ WHOcausality_assessment.pdf. Accessed October 11, 2021. 
32. Sarayani A, Cicali B, Henriksen CH, Brown JD. Safety signals for QT prolongation or torsades de pointes associated with azithromycin with or without chloroquine or hydroxychloroquine. Res Soc Adm Pharm. 2021;17:483. doi:10.1016/j.sapharm.2020.04.016

33. Yang TH, Chou C-Y, Yang Y-F, et al. Systematic review and meta-analysis of the effectiveness and safety of hydroxychloroquine in treating COVID-19 patients. J Chin Med Assoc. 2021;84:233-241. doi:10.1097/JCMA.0000000000000425

34. Mittal N, Mittal R, Gupta MC, et al. Systematic review and meta-analysis of efficacy and safety of hydroxychloroquine and chloroquine in the treatment of COVID-19. J Family Med Prim Care. 2021;10(6):2126. doi:10.4103/jfmpc.jfmpc_2338_20

35. Chen C, Pan K, Wu B, et al. Safety of hydroxychloroquine in COVID-19 and other diseases: a systematic review and meta-analysis of 53 randomized trials. Eur $J$ Clin Pharmacol. 2021;77(1):13-24. doi:10.1007/s00228-020-02962-5

36. Bustos MDG, Gay F, Diquet B, Thomare P, Warot D. The pharmacokinetics and electrocardiographic effects of chloroquine in healthy subjects. Trop Med Parasitol. 1994;45:83-86.

37. Chen CY, Wang FL, Lin CC. Chronic hydroxychloroquine use associated with QT prolongation and refractory ventricular arrhythmia. Clin Toxicol. 2006;44:173-175. doi:10.1080/15563650500514558

38. Hsia BC, Greige N, Quiroz JA, et al. QT prolongation in a diverse, urban population of COVID-19 patients treated with hydroxychloroquine, chloroquine, or azithromycin. J Interv Card Electrophysiol. 2020;59(2):337-345. doi:10.1007/s10840-020-00822-x

39. Howard PA. Azithromycin-induced proarrhythmia and cardiovascular death. Ann Pharmacother. 2013;47(11):1547-1551. doi:10.1177/ 1060028013504905

40. Maisch NM, Kochupurackal JG, Sin J. Azithromycin and the risk of cardiovascular complications. J Pharm Pract. 2014;27(5):496-500. doi: $10.1177 / 0897190013516503$

41. Zhang M, Xie M, Li S, et al. Electrophysiologic studies on the risks and potential mechanism underlying the proarrhythmic nature of azithromycin. Cardiovasc Toxicol. 2017;17(4):434-440. doi:10.1007/s12012-017-9401-7

42. Choi Y, Lim HS, Chung D, Choi JG, Yoon D. Risk evaluation of azithromycin-induced QT prolongation in real-world practice. Biomed Res Int. 2018;2018:1574806.

43. Bhatla A, Mayer MM, Adusumalli S, et al. COVID-19 and cardiac arrhythmias. Heart Rhythm. 2020;17(9):1439-1444. doi:10.1016/j. hrthm.2020.06.016

44. Kunutsor SK, Laukkanen JA. Renal complications in COVID-19: a systematic review and meta-analysis. Ann Med. 2020;52:1-9. doi: $10.1080 / 07853890.2020 .1790643$
45. Rosenberg ES, Dufort EM, Udo T, et al. Association of treatment with hydroxychloroquine or azithromycin with in-hospital mortality in patients with COVID-19 in New York State. JAMA. 2020;323 (24):2493-2502. doi:10.1001/jama.2020.8630

46. Cevik M, Tate M, Lloyd O, et al. SARS-CoV-2, SARS-CoV, and MERS-CoV viral load dynamics, duration of viral shedding, and infectiousness: a systematic review and meta-analysis. Lancet Microbe. 2021;2(1):e13-e22. doi:10.1016/S2666-5247(20)30172-5

47. Abrahim SA, Tessema M, Defar A, et al. Time to recovery and its predictors among adults hospitalized with COVID-19: a prospective cohort study in Ethiopia. PLoS One. 2020;15:e244269.

48. Chen X, Zhu B, Hong W, et al. Associations of clinical characteristics and treatment regimens with the duration of viral RNA shedding in patients with COVID-19. Int $J$ Infect Dis. 2020;98:252-260. doi:10.1016/j.ijid.2020.06.091

49. Hu Z, Song C, Xu C, et al. Clinical characteristics of 24 asymptomatic infections with COVID-19 screened among close contacts in Nanjing, China. Sci China Life Sci. 2020;63(5):706-711. doi:10.1007/s11427-020-1661-4

50. Sakurai A, Sasaki T, Kato S, et al. Natural history of asymptomatic SARS-CoV-2 infection. $N$ Engl $J$ Med. 2020;383:885-886. doi:10.1056/NEJMc2013020

51. Yang R, Gui X, Xiong Y. Comparison of clinical characteristics of patients with asymptomatic vs symptomatic coronavirus disease 2019 in Wuhan, China. JAMA Netw open. 2020;3:e2010182. doi:10.1001/ jamanetworkopen.2020.10182

52. Xiao T, Wang Y, Yuan J, et al. Early viral clearance and antibody kinetics of COVID-19 among asymptomatic carriers. medRxiv. 2020;2020. doi:10.1101/2020.04.28.20083139

53. Han MS, Seong M-W, Kim N, et al. Viral RNA load in mildly symptomatic and asymptomatic children with COVID-19, Seoul, South Korea. Emerg Infect Dis. 2020;26(10):2497-2499. doi:10.3201/eid2610.202449

54. Jones JK. Determining causation from case reports. In: Strom B, editor. Pharmacoepidemiology. NY: John Wiley \& Sons; 2007:555-570.

55. Arimone Y, Bégaud B, Miremont-Salamé G, et al. Agreement of expert judgment in causality assessment of adverse drug reactions. Eur J Clin Pharmacol. 2005;61(3):169-173. doi:10.1007/s00228004-0869-2
Therapeutics and Clinical Risk Management

\section{Publish your work in this journal}

Therapeutics and Clinical Risk Management is an international, peerreviewed journal of clinical therapeutics and risk management, focusing on concise rapid reporting of clinical studies in all therapeutic areas, outcomes, safety, and programs for the effective, safe, and sustained use of medicines. This journal is indexed on PubMed Central, CAS,

\section{Dovepress}

EMBase, Scopus and the Elsevier Bibliographic databases. The manuscript management system is completely online and includes a very quick and fair peer-review system, which is all easy to use. Visit http://www.dovepress.com/testimonials.php to read real quotes from published authors. 